\title{
LES FIGURES SOCIALES DE L'USAGER DE LA PrEP AU PRISME DE L'ANALYSE DE TEMOIGNAGES EN LIGNE
}

\author{
CÉCILE LORIATO \\ Université Paris-Est Créteil Val-de-Marne
}

\section{RÉSUMÉ}

En prenant pour objet d'analyse le témoignage en ligne, cette contribution propose d'éclairer les figures sociales de l'usager de la PrEP, nouvel outil de prévention du VIH, afin de questionner les représentations de la sexualité gay, telles qu'elles apparaissent dans les interactions entre les participants à un groupe de discussion Facebook dédié à la PrEP. Les témoignages ont été analysés dans une perspective diachronique dans le cadre d'une ethnographie en ligne pour laquelle ont été mobilisées plusieurs méthodes : l'observation et l'analyse du dispositif sociotechnique, des entretiens semi-directifs, une analyse qualitative des interactions, ainsi qu'une analyse quantitative de la participation. L'analyse des récits de soi, en tant qu'objets contraints par un dispositif sociotechnique institutionnalisé et mouvants dans l'interaction, a permis de mettre en évidence des figures sociales de l'usager de la PrEP, qui se constituent par le croisement de la nature du comportement (conforme ou non-conforme à la norme) avec les réactions qu'il suscite (c'est-à-dire en fonction de la perception qu'en ont les participants au groupe). Ainsi, ont émergé la figure du "prepeur raisonnable", celle du "bon prepeur" en opposition à la figure du "mauvais prepeur", puis celle du "prepeur responsable".

Mots clés : Témoignage en ligne. Prévention du VIH. Homosexualité. PrEP

\section{ABSTRACT THE SOCIAL FIGURES OF THE USER PrEP UNDER THE PRISM OF ON-LINE WITNESSES ANALYSIS}

By taking as an object of analysis the online testimony, this contribution suggests enlightening social figures of the user of PrEP, a new mean of HIV prevention, in order to question the representations of gay sexuality such as they appear in the interactions between participants to a Facebook group dedicated to PrEP. The testimonies were analyzed in a diachronic perspective using an online ethnography 
for which several methods have been mobilized: the observation and the sociotechnique analysis of the Facebook group, semi-directive interviews, a qualitative analysis of the interactions, as well as a quantitative analysis of the participation. The analysis of self-stories, as objects compelled by an institutionalized apparatus and moving along with the interactions, was useful to identify social figures of the PrEP user, which are formed by the meeting of the behavior (consistent or not with the norm) with the reactions it induces (i.e., based on the perception of the others participants). Thus, have emerged the figure of the "reasonable PrEP user", the one of the "good PrEP user" in opposition to the "bad PrEP user", and then the figure of the "responsible PrEP user".

Keywords: Online testimony. HIV prevention. Homosexuality. PrEP.

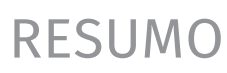

\section{AS FIGURAS SOCIAIS DO USUÁRIO PREP SOB O PRISMA DA ANÁLISE DE TESTEMUNHOS ON-LINE}

Tomando como objeto de análise o testemunho on-line, esta contribuição propõe focalizar as figuras sociais do usuário da PrEP uma nova ferramenta de prevenção do HIV - a fim de questionar as representações da sexualidade gay, tal como elas aparecem nas interações entre os participantes de um grupo de discussão num facebook dedicado à PrEP. Os testemunhos foram analisados numa perspectiva diacrônica, no contexto de uma etnografia on-line, mobilizando-se vários métodos: observação e análise do dispositivo sociotécnico, entrevistas semiestruturadas, análise qualitativa das interações, assim como uma análise quantitativa da participação. A análise das narrativas de si, consideradas como objetos produzidos por um dispositivo sociotécnico institucionalizado e movimentos na interação, permitiu destacar figuras sociais do usuário da PrEP, que se constituem pelo entrecruzamento da natureza do comportamento (de acordo ou não com a norma) e das reações que ele suscita (isto é, em função da percepção que têm os participantes do grupo). Assim, emergiram a figura do "preper razoável", a do "bom preper", em oposição à figura do "mau preper" e ainda a do "preper responsável". Palavras-chave: Testemunho online. Prevenção do HIV. Homossexualidade. PrEP.

"Dans la sérophobie c'est souvent les gens qui ont peur de se faire contaminer. Du coup ils rejettent. La prépophobie je suis pas sûr que ce soit la peur. Le terme est peut-être pas très bien choisi. Je pense que c'est plus un jugement mo- ral sur le choix des gens qui prennent la PrEP. Et souvent ces gens là n'ont pas de doute sur l'efficacité de la PrEP. S'ils sont contre la PrEP ils le sont souvent pour plein d'autres raisons. Je pense que ça les renvoie à une sexualité hors 
contrôle. Je pense qu'ils ont peur de la perte de contrôle de leur propre sexualité. Et du coup ils rejettent le fait que certains puissent être complètement libérés des freins qui les empêchent de s'épanouir sexuellement. Il y a plein de gens pour qui la PrEP c'est la porte ouverte à n'importe quoi. Sauf que ça veut dire quoi "faire n'importe quoi »? C'est du jugement moral de base. Pour moi c'est la même chose que les gens qui rejettent les gays efféminés ou la gaypride, qui pensent que l'homosexualité sera plus acceptée si on était tout propre, tout gentil, et qu'on rentrait dans un espèce de moule hétéronormé, monogame, etc. Du coup la PrEP c'est une façon d'avoir une sexualité sans préservatif, donc ça renvoie aux partouzes, etc. Ce qui n'est pas forcément vrai. Il y a plein de gens qui prennent la PrEP et qui ne sont pas comme ça. "

Entretien avec un membre de PrEP'Dial, janvier 2018.

\section{Introduction}

En septembre 2015, AIDES, une des associations les plus importantes de lutte contre le VIH/sida, crée un groupe de discussion numérique, au moyen de la plateforme Facebook. Ce groupe, nommé PrEP'Dial, est dédié à un nouvel outil de prévention du VIH, la prophylaxie pré-exposition (PrEP). C'est une bithérapie ${ }^{1}$ sous forme de comprimé de $\operatorname{Truvada}^{\circledR}$ ou, depuis 2017, d'un générique, qui peut être pris en continu, c'est-à-dire quotidiennement, ou à la demande, avant et après un rapport sexuel à risque. Cet outil est recommandé pour les personnes séronégatives à fort risque d'acquisition du VIH par voie sexuelle, soit les hommes ayant des rapports sexuels avec des hommes (HSH) et les personnes trans ${ }^{2}$. Le groupe PrEP'Dial permet d'une part de faire connaître la PrEP à des individus qui pourraient en avoir

1 C'est-à-dire une combinaison de deux antirétroviraux hautement actifs contre le VIH, le Ténofovir et l'Emtricitabine.

2 Source : http://ansm.sante.fr/S-informer/Points-d-information-Points-d-information/L-ANSM-etablit-la-RTU-de-Truvada-dans-la-prophylaxie-pre-exposition-auVIH-Point-d-information (Consulté le 23 janvier 2016) besoin, d'autre part de mettre à disposition de toute personne intéressée par ce nouvel outil un espace d'échange et de partage des expériences individuelles de prévention et, plus particulièrement, de l'utilisation de la PrEP. Ainsi, le témoignage est une forme importante de participation dans PrEP'Dial. Comme tout récit de soi, le témoignage en ligne est

«[...] pris dans une tension entre assujettissement et subjectivation, entre bio-politique comme gouvernement des corps et de la vie, comme pouvoir de " conduire les conduites» (Foucault), et auto-bio-graphie comme effort du sujet de se constituer lui-même et de donner une forme à son expérience. " (NIEWIADOMSKI et Delory-MOMBERGER, 2013, p. 45)

Cette ambivalence se manifeste par un dispositif sociotechnique contraignant tout en offrant au participant la possibilité de se raconter. L'exposition de soi sur les réseaux sociaux est liée à une demande de reconnaissance par autrui d'une singularité identitaire (GRANJON, 2012). Le témoignage, comme mise en scène de soi, résulte de « pratiques de soi-même ", qui favorisent le processus de réalisation individuelle, et de " pratiques de soi à travers autrui », qui s'organisent par « le prisme de procédures de reconnaissance »(DENOUËL, 2011). Que ce soit en ligne ou hors ligne, le témoin oriente et développe son récit selon la perception qu'il a du contexte, de ses interlocuteurs : il témoigne conjointement pour lui-même et pour l'autre, il " prend à témoin » (CORDONNIER, 2015, p. 11). Le témoignage sur un groupe de discussion en ligne a ceci de particulier que le travail sur soi se fait dans l'interaction avec les autres, cadrée par le dispositif sociotechnique, qui impose en quelque sorte un contrat tacite entre les participants. L'asynchronicité et la persistance des messages, la structure des échanges, ainsi que le rôle de AIDES en tant qu'animatrice et modératrice du groupe 
conditionnent la production et la réception du récit, créant une sorte de dépendance au dispositif. Celle-ci s'observe plus particulièrement dans la double réflexivité du témoin : celle, assumée, dont il fait preuve en écrivant son témoignage et celle, subie, qui s'impose dans les interactions cadrées par le dispositif, le menant parfois à la modification de son récit. Dans notre recherche doctorale en cours sur l'appropriation de la PrEP, nous nous intéressons au témoignage au sein de PrEP'Dial en ce qu'il publicise des manières de voir et des manières de faire individuelles, mais aussi des problèmes de société plus généraux, par exemple la stigmatisation des pratiques sexuelles non protégées par un préservatif et, plus particulièrement, la stigmatisation d'un certain mode de vie attribué à des hommes homosexuels. En effet, la PrEP arrive dans un contexte fortement marqué par une recrudescence des comportements à risque et une baisse de l'utilisation du préservatif, notamment chez les HSH (VELTER et al., 2011). Ainsi, dès le recrutement pour l'essai ANRS Ipergay ${ }^{3}$ débuté en 2012, il est admis que la PrEP n'est pas destinée à toute la population, mais à un public $d$ ' " hommes faillibles ", selon les termes de P. Ricœur (TRACHMAN et GIRARD, 2018 , p. 22), soit « d'individus à la fois incapables de se protéger et capables de suivre un schéma de traitement complexe et de rationaliser leur sexualité » (TRACHMAN et GIRARD, 2018 , p. 22). Tout en étant significative de la diversification des moyens de prévention, l'utilisation de la PrEP s'inscrit donc dans des débats moraux autour de l'homosexualité, du VIH et du risque (GIRARD, 2016), ravivant des controverses sur la désinhibition sexuelle et les prises de risque délibérées, comme le

3 L'essai ANRS Ipergay débute en 2012 en France et au Québec. En 2015, il prouve l'efficacité de la PrEP à la demande (c'est-à-dire avant et après un rapport sexuel) pour les hommes ayant des rapports sexuels avec d'autres hommes (HSH). bareback dans les années 1990. Ce terme anglais signifiant « monter à cheval sans selle » est utilisé par les homosexuels pour désigner des pratiques sexuelles non protégées (GIRARD, 2013). Ces débats mettent en opposition les "bons » homosexuels, qui se protègent mais rencontrent parfois des difficultés à utiliser un préservatif, et les « mauvais » homosexuels, qui ne se protègent pas, de manière délibérée. Ainsi, bien que la PrEP ait été autorisée en 2016 dans le cadre d'une recommandation temporaire d'utilisation (RTU) puis qu'elle ait fait l'objet d'une autorisation de mise sur le marché (AMM) en 2017, son introduction a pu paraître complexe à articuler avec d'autres stratégies de prévention institutionnalisées et notamment, la norme de l'utilisation du préservatif. Dans une perspective interactionniste (MEAD, 1963; GOFFMAN, 1974, 1987, 1991; BECKER, 1985), on considère que les témoignages qui circulent au sein de PrEP'Dial permettent un échange de significations entre des participants réflexifs dont les interactions vont permettre une relecture des normes de prévention, et du monde social de manière générale, à travers « des interprétations mutuelles suscitant un ajustement des acteurs les uns par rapport aux autres » (LE BRETON, 2016 (2004)). En prenant pour objet d'analyse le témoignage en ligne, cette contribution propose d'éclairer les figures sociales de l'usager de la PrEP afin de questionner les représentations de la sexualité gay, entre déviance et normativité, telles qu'elles apparaissent dans les interactions entre les participants à PrEP'Dial. La déviance est entendue ici comme l'ensemble des " comportements qui transgressent des normes acceptées par tel groupe social ou par telle institution » (BECKER, 1985, p. 9). En premier lieu, on présentera la méthodologie, puis on s'attachera à caractériser le groupe de discussion ainsi que la dynamique de la participation. On exposera ensuite les différentes 
fonctions du témoignage sur PrEP'Dial avant de décrire les figures sociales de l'utilisateur de la PrEP.

\section{Méthodologie : une ethnographie} d'un espace de discussion en ligne sur la prévention du VIH/sida

Ces récits de soi ont été recueillis à partir d'une ethnographie du groupe de discussion Facebook PrEP'Dial, inspirée de méthodes d'analyse du web (AKRICH et MEADEL, 2007, 2009 ; PASTINELLI, 2011 ; AKRICH, 2012 ; JOUËT et LE CAROFF, 2013 ; HINE, 2015 ; LE CAROFF, 2015 ; MARCOCCIA, 2016), réalisée dans le cadre d'une recherche doctorale en cours. La démarche ethnographique ne conçoit pas le terrain d'enquête uniquement comme un réservoir de données mais également comme l'objet de la recherche. L'ethnographie en ligne est « fondée sur l'étude descriptive et analytique des écrits numériques des internautes, qui demeurent invisibles dans le cours de leurs interactions » (JOUËT et LE CAROFF, 2013, p. 147). Pour comprendre le sens que donnent les acteurs à ce qu'ils disent faire de leur prévention, il faut donc également s'intéresser à d'autres données que celles contenues dans les écrits. Pour réaliser cette ethnographie, nous avons eu recours à plusieurs méthodes : l'observation, l'élaboration d'une grille d'observation du dispositif sociotechnique inspirée de celle de J. Jouët et C. Le Caroff (2013), ainsi que des entretiens semi-directifs menés avec douze membres du groupe qui se sont portés volontaires. Nous avons également effectué une analyse quantitative de la participation à partir de méthodes manuelles et de tableurs Excel afin d'établir une typologie des contributeurs et d'observer la dynamique de participation. Enfin, nous avons effectué une analyse thématique et qualitative des interactions afin de saisir les justifications des membres de PrEP'Dial quant à leur prévention. Au total, nous avons sélectionné et analysé 349 publications et 6417 commentaires, publiés par 755 participants actifs sur un nombre de membres s'élevant à 8834 à la clôture de notre échantillon. La période d'analyse s'étend de la création du groupe en septembre 2015 à novembre 2017, soit 27 mois. Dans une perspective diachronique, les données ont ensuite été analysées en relation avec trois séquences temporelles : celle se situant avant la RTU (septembre 2015-décembre2015), celle de la RTU (janvier 2016-décembre 2016) et celle commençant avec l'AMM jusqu'à la clôture de l'échantillon (janvier 2017-novembre 2017).

\section{PrEP’Dial, une arène semi-publique de discussion sur la prévention du $\mathrm{VIH} /$ sida}

PrEP'Dial est un groupe support. Il a pour objectif d'accompagner le développement de la PrEP par, pour et avec les utilisateurs de la PrEP. Un groupe de support virtuel est un espace en ligne de discussion et d'échange d'expériences et d'informations rassemblant des individus partageant un intérêt commun pour le sujet auquel le groupe est dédié (ROJAS CASTRO, 2009). Selon ses concepteurs associatifs, un tel espace, libéré des contraintes géographiques et horaires, permet à ces individus de parler de leur santé sexuelle, a priori sans crainte d'un jugement stigmatisant. Toutefois, l'observation du groupe a permis d'identifier des éléments de débat et de fonctionnement qui caractérisent en général une arène publique. Selon la définition de N. Dodier (1999, p. 109), une arène est

«[...] un dispositif visant à mettre en relation des locuteurs et des audiences auxquelles ils s'adressent. Quatre éléments la caractérisent : 1. Des conditions régissant l'entrée des locuteurs et ce sur quoi ils peuvent s'exprimer; 2 . Des 
conditions relatives au mode de confrontation entre locuteurs, ou entre locuteurs et audience ; 3. Des supports d'inscription des discours produits (papier, films, vidéos, supports électroniques, etc); 4. Des conditions d'accès pour les audiences (grand public, membres d'une organisation, spécialistes [...] $»$.

PrEP'Dial est caractérisé par ces quatre éléments. En premier lieu, le groupe est «fermé ", c'est-à-dire qu'il n'est accessible qu'aux membres et l'acceptation au sein du groupe est à la discrétion des administrateurs. De plus, « chaque arène dispose de sa propre culture de débat, qui implique des manières spécifiques de mettre en discussion des sujets et de considérer comme valides les arguments échangés » (BADOUARD, MABI et MONNOYER-SMITH, 2016, p. 11). Dans une analyse de listes de discussion, $M$. Akrich et $C$. Méadel (2007, p. 146) remarquent la constitution d'une " philosophie » propre à chaque liste, délimitant un espace de débat dans lequel certaines questions peuvent être discutées et d'autres non. Ainsi, on constate que PrEP'Dial est une arène institutionnalisée par AIDES, qui cadre le débat de manière " philosophique » et technique. Les objectifs du groupe, tout comme ses règles de fonctionnement et de participation, sont définis dans une " charte ", c'est-à-dire dans un texte visant à cadrer les interventions. De plus, la participation active de militants de AIDES à la diffusion d'informations et de documentation souvent élaborée par l'association contribue à la circulation d'une grammaire qui lui est spécifique. Cependant, PrEP'Dial ne vise pas particulièrement à mettre en relation des locuteurs et des audiences auxquelles ils s'adressent. Le groupe n'a pas vocation à publiciser un problème. Ainsi, il est loin de la définition que donne D. Céfaï (2016, p. 38) d'une arène publique « dont les acteurs visent des biens publics, se réfèrent à l'intérêt public, définissent leurs problèmes comme publics et sentent, agissent et parlent en conséquence ". Le débat a lieu dans d'autres arènes, accessibles uniquement aux experts, aux institutions de santé publique et aux associations. Cependant, si PrEP'Dial n'a pas pour objectif principal la publicisation de la PrEP, le groupe donne à voir les difficultés d'accès à ce nouvel outil (lenteur d'action des pouvoirs publics, manque d'écoute et de compréhension des professionnels de santé, manque d'information, stigmatisation, etc), qui font l'objet de discussions.

Par ailleurs, les discussions révèlent des manières de voir différentes, c'est-à-dire des grilles de perception et de modes d'interprétation des événements différentes (CARBOU, 2015), qui se manifestent en particulier dans des récits personnels, quotidiens et intimes qui circulent au sein du groupe. En cela, PrEP'Dial s'apparente à un espace privé / public, défini par D. Mehl (2003, p. 501) dans une analyse de la télévision de l'intimité contemporaine comme

«[..] un espace de monstration d'une diversité d'expériences singulières, essentiellement sur le mode du témoignage personnel, cette dynamique de publicisation ouvrant à des délibérations reformulées dans le cadre de colloques privés. Il ne signe ni la mort de l'espace public, ni l'aphonie de l'espace privé, mais une articulation particulière où le pluralisme des témoignages se présente comme le garant virtuel de l'énoncé de points de vue discordants et donc de l'hypothèse d'un débat [...] ».

Toutefois, le format du groupe Facebook de discussion étant différent à bien des égards de celui d'une émission de télévision, il convient de relativiser ce rapprochement pour l'analyse de PrEP'Dial. Par exemple, le participant d'un show télévisuel est recruté alors que celui de PrEP'Dial, bien que son acceptation ait été soumise à la validation des administrateurs, peut faire part de son expérience sans y être spé- 
cifiquement invité - de fait, son acceptation au sein du groupe constitue une invitation à témoigner. Cependant, D. Mehl montre que se noue une collaboration tacite entre l'émetteur du témoignage et le spectateur qui le reçoit : le premier publicise son cas particulier qui devient général en permettant au second de se l'approprier. Sur PrEP'Dial, le spectateur est un participant passif, qui endosse simplement le rôle de lecteur, mais peut être également actif, en choisissant d'interagir avec le témoin en devenant, souvent, témoin à son tour.

\section{Esquisse d'une typologie des participants}

Au cours de notre recherche doctorale, nous avons réalisé une typologie des participants, à partir de plusieurs critères de participation : le nombre de contributions, la régularité de la participation, la durée d'inscription au groupe et le type de contribution. Ont ainsi émergé six catégories de participants : les hyperactifs, les assidus, les intermittents, les éphémères, les discrets, les invisibles. Les contributeurs « hyperactifs » (HYPE) représentent moins de 1\% de l'échantillon. Ils ont une participation très élevée et très régulière. Ce sont tous des membres de AIDES, salariés et/ou volontaires, inscrits depuis la création du groupe. Leur participation consiste à diffuser de l'information, à proposer des actions, ainsi qu'à répondre aux questions pratiques et techniques sur la PrEP. Ils utilisent le témoignage de leurs expériences en tant qu'utilisateur de la PrEP et/ou militant pour promouvoir ce nouvel outil ainsi que pour répondre à des besoins d'information. Cependant, davantage disposés à soutenir les autres participants qu'à se mettre en avant, ils ne font pas du témoignage un usage privilégié. Les contributeurs " assidus » (ASSI) représentent moins de $3 \%$ de l'échantillon. Ils sont caractérisés par la régularité de leur participation, tout en présentant un niveau de participation variable. De plus, ils sont toujours inscrits au groupe à la fermeture de l'échantillon. S'ils diffusent des informations, répondent aux questions techniques et pratiques sur la PrEP et émettent des propositions, le récit de leurs expériences est présent dans la plupart de leurs contributions, que ce soit de manière détaillée ou simplement pour illustrer un argument. La moitié appartient au milieu de la santé et/ ou de la lutte contre le VIH/sida. Les contributeurs « intermittents » (INTE) représentent 4\% de l'échantillon. Ils participent beaucoup mais de manière irrégulière, tout en restant inscrits au groupe même lorsqu'ils ne contribuent plus aux discussions. Les types de contribution sont divers, allant de l'appel au témoignage et de la demande d'information, à la réponse à des questions pratiques et techniques, en passant par une participation active aux débats sur la PrEP. Un tiers appartient au milieu de la santé et/ou de la lutte contre le VIH/sida. Les contributeurs « éphémères » (EPHE) représentent $10 \%$ de l'échantillon. Ils quittent le groupe peu de temps après s'y être inscrits, présentent un niveau de participation variable, et critiquent la PrEP ainsi que le fonctionnement du groupe. Leur recours au témoignage est moindre et semble davantage exprimer leur expérience du groupe que de la prévention. Ils sont généralement extérieurs au milieu de la santé et/ou de la lutte contre le VIH/sida et privilégient l'anonymat de leurs profils Facebook. Les contributeurs « discrets » (DISC) représentent $75 \%$ de l'échantillon. Ils participent peu, une unique fois ou de manière irrégulière, tout en restant inscrits durablement au groupe. De manière générale, ils socialisent, manifestent leur soutien et leur reconnaissance aux autres participants. Chez eux, le témoignage se manifeste généralement peu sous une forme détaillée, mais plutôt par des marques d'identification au récit de leur interlocuteur, telles 
que « il m'est arrivé la même chose ", parfois suivies de quelques détails sur la situation en question. La plupart sont extérieurs au milieu de la santé, mais $14 \%$ d'entre eux sont militants ou salariés chez AIDES, au sein d'autres organismes de lutte contre le VIH/sida ou sont des professionnels de la santé. Les participants « invisibles » (INVI) sont ceux dont on n'a aucune trace de la participation sur la période analysée. Ils sont inscrits au groupe mais n'y participent pas activement. Ainsi, à la fin du mois de novembre 2017, sur 8834 inscrits 2843 étaient actifs selon une estimation statistique fournie par Facebook, le terme « actif » comprenant tout participant ayant réagi au moins une fois à une publication au cours du mois. P. Flichy constate qu'au sein d'une communauté virtuelle « ceux qui s'expriment sont rares et que la grande majorité observe le débat (lit les messages) sans intervenir de façon active dans la discussion » (FLICHY, 2001, p. 111). D'autres encore sont membres mais ont fait le choix de ne pas recevoir de notifications et vont rarement lire les actualités du groupe. Ils ont été ajoutés par des amis ou ont fait une demande d'inscription par curiosité, ou parce qu'ils recherchaient une information à un moment donné, mais n'utilisent pas le groupe comme un support quotidien. Une catégorie « autres » (code: AUTR) regroupe les membres dont l'inscription proche de la clôture de notre échantillon ne permet pas de qualifier la participation. Par manque de place, nous ne pouvons développer davantage ici cette typologie. Elle n'en est pas moins importante pour l'analyse des témoignages car elle nous donne un aperçu de la dynamique de participation et de la socialisation des participants dans le milieu de la santé et de la lutte contre le VIH/sida. Si l'on considère que «lors de la production du témoignage, le témoin constitue et mobilise ses propres catégories d'interprétation du monde » (CORDONNIER, 2015, p. 14), alors cette connaissance des participants, bien que partielle, aide à mieux comprendre qui écrit quoi.

\section{Les différentes fonctions du témoignage au sein de PrEP'Dial}

« S'il tend à valoriser la singularité des subjectivités, le témoignage de l'expression citoyenne s'ouvre cependant à la montée en généralité. Il fait alors du récit qu'il porte et rend public une expérience possiblement partagée/partageable par d'autres » (DENOUËL, 2011, p. 78).

Ainsi, le témoignage peut revêtir plusieurs fonctions. Nous en avons identifié trois au sein de PrEP'Dial : informer, sensibiliser et revendiquer. Le témoignage peut être une source d'information : participer au groupe de discussion " serait un moyen de s'informer au travers de l'expérience d'autres malades, de partager son vécu, de se rassurer sur son avenir » (PAGANELLI et CLAVIER, 2011, p. 43). Le témoignage peut alors servir à documenter des situations, des cas de figure. Il s'agit de recueillir des expériences dans un but personnel - un individu souhaitant comparer son expérience à celles des autres utilisateurs -, ou avec un objectif collectif - par exemple recueillir les expériences des utilisateurs pour améliorer les services de consultation ou documenter les différents effets secondaires. Ensuite, le témoignage peut également sensibiliser à l'utilisation de la PrEP. Il peut s'agir de liens hypertextes vers des dispositifs de communication ou des dispositifs médiatiques. Ils mettent en scène des utilisateurs de la PrEP, membres de PrEP'Dial, qui font le récit de leurs expériences à une audience plus large et plus composite que celle du groupe, dans un cadre institutionnel comme un reportage dans un journal télévisé ou encore un support de communication produit par AIDES. Ce témoignage sert en premier lieu à attirer l'attention sur la PrEP à l'extérieur du groupe. Au sein du groupe, ces témoignages 
légitimés par le dispositif qui les diffuse font échos aux expériences personnelles des autres participants. De plus, le récit de soi peut également revêtir cette fonction de sensibilisation lorsqu'il s'agit d'attirer l'attention sur un problème systémique, par exemple le manque d'information des personnels médicaux. Enfin, le témoignage peut servir de revendication. Il peut s'agir de revendiquer le droit d'accéder à la PrEP gratuitement et en toute sécurité, comme avant la RTU et l'AMM. Ce type de témoignage vise à interpeler les décideurs. Il est, en général, intégré dans un dispositif médiatique. Il apparaît sur PrEP'Dial sous forme de lien vers le dispositif en question. Ainsi, il n'est pas le fruit du groupe mais permet de diffuser les actions menées en faveur de la PrEP. Par exemple, en octobre 2015, un administrateur publie un lien vers une tribune publiée sur yagg.com, un média LGBT en ligne, écrite par un journaliste revendiquant l'autorisation de la PrEP. Ce dernier justifie sa réclamation en faisant le récit de ses expériences sexuelles ${ }^{4}$. Il peut également s'agir de témoignages revendiquant l'utilisation de la PrEP, qui ont souvent une visée militante. Ils peuvent prendre plusieurs formes, notamment celle de photos mettant en scène le contributeur prenant son comprimé, ou encore de textes expliquant le choix de l'utilisation de la PrEP, dénonçant souvent les discriminations à l'égard de ses utilisateurs et plus largement, des personnes vivant avec le VIH (PVVIH). Ce type de témoignage a pour but de libérer la parole sur la PrEP et d'encourager les utilisateurs à affirmer leurs choix de prévention, sans en avoir honte.

Quelle que soit leur fonction première, ces témoignages présentent des degrés de singularité des subjectivités différents. Ils peuvent être sobres et aller à l'essentiel, tout comme

4 PATINIER Jérémie. Ceci est un appel d'urgence : je veux la PrEP parce que je veux rester séronégatif. Yagg. com, 14 octobre 2015, disponible sur http:/ / www.yagg. com. ils peuvent être chargés d'émotion et riches en détails. Le témoignage est alors la mise en scène de soi par excellence pour certains participants, qui semblent considérer PrEP'Dial comme un espace personnel au sein duquel ils feraient part de leurs états d'âme, de leurs expériences et de leurs opinions à un public d'internautes, sur le modèle du blog par exemple (PAGANELLI et CLAVIER, 2011, p. 48). Le témoignage revêt alors une forte valeur identificatoire, dont témoignent les réactions d'approbation souvent suivies du récit des expériences des interlocuteurs : ainsi les interactions valident et légitiment le vécu. Si ces témoignages sont utiles aux participants au groupe, ils constituent également une source d'information pour AIDES, qui peut ainsi identifier certains besoins et améliorer ses services.

\section{Essai de typologie des figures sociales de l'usager de la PrEP}

À partir de six fils de discussion extraits de PrEP'Dial, il s'agit ici, dans une perspective diachronique, d'observer comment les participants définissent leur utilisation de ce nouvel outil de prévention au cours des différentes séquences du processus de reconnaissance de la PrEP5.

\section{Avant la RTU, le « prepeur ${ }^{6}$ » raisonnable}

5 Guide de lecture des extraits de PrEP'Dial : les réponses à la publication sont alignées sous celle-ci, au même niveau. Les réponses à un commentaire sont situées à $1 \mathrm{~cm}$ en retrait vers la droite au-dessous de celui-ci. Les points de suspension entre parenthèses signifient que d'autres commentaires complétaient le fil de discussion mais nous avons choisi de les couper, d'une part car ils nous semblaient peu pertinents pour la compréhension (il s'agissait en général de marques de socialisation), d'autre part par manque de place. Les participants sont anonymisés par un code indiquant le type de participant accompagné d'un numéro.

6 Le terme « prepeur » correspond à l'appellation que se donnent les usagers de la PrEP entre eux. 


\section{Encadré 1 - Extrait de PrEP'Dial, septembre 2015}

INTE001 QUESTION : Et nous en France, à quand un collage d'affiches sauvage dans le Marais ??? (lien vers un article de pinknews.co.uk : "Shock poster campaign tells gays to $« f * * *$ raw - PrEP works ». Posters encouraging people to have sex without condoms have appeared in Melbourne »)

HYPE001 Je suis à fond pour un collage sauvage pro-PREP dans le marais mais je suis pas sûr de la pertinence de ce message $\ddot{\cup}$ Inventons notre propre message!

INTE001 Il va de soi qu'en France, il y a des enjeux quant à l'accès à la PREP... Il faut être prudent.

\section{[...]}

DISC011 et pourquoi pas une traduction littérale : "Vous pouvez baiser Bareback - la PrEP fonctionne - Stop au VIH »

INTE001 Non... je ne me considère pas comme un barebacker en étant utilisateur de Prep ... et je pense que nous risquons dans ce cas de passer pour des terroristes....

HYPE001 Pareil je suis prepeur et fais du No Kpote souvent mais je ne me considère pas comme barebackeur.

\section{[...]}

DISC011 le terme raw est difficilement traduisible ...

INTE001 Oui, certains parlent de bio... Mais sous Truvada, j'avoue avoir du mal à me sentir viande bio ...

\section{[...]}

INTE001 Pour ma part, je sais que j'ai très peu d'activités militantes, mais qu'il me semble important de revendiquer dans les apps de rencontre que je suis sous prep. Ça suscite des interrogations, des peurs... Mais il me semble nécessaire d'investir l'espace public. Pour les autres, mais aussi pour nous, les prepeurs... Nous sommes, je trouve, parfois un peu seuls...

HYPE001 En même temps ce n'est pas à nous de dire comment baiser. On a tous ici souffert de l'injonction à la capote obligatoire, ce n'est pas pour faire l'inverse et faire des injonctions à baiser sans capote (parfois ou tout le temps) il y a la PrEP pour se protéger du VIH...

INTE003 Je suis anglophone, donc je me sens obliger de parler de ces deux mots, raw et bareback. Pour clarifier, il faut que vous comprenez les origines de ces deux mots. Au début, «bareback " était un mot assez classe et noble qui signifier les dames qui montent au cheval sans selle et plus tard, utiliser par les cowboys masculins et viriles dans les films et pendant les rodéos. C'était une mot qui a existé depuis des années. Le terme Raw est vulgaire, limite dégoutant. Raw c'est cru. Raw c'est une plaie ouverte. Pour moi, la prep est ni Bareback, ni Raw. La prep est une méthode en plus pour stopper la transmission du virus. Pour moi, bareback et raw sont des termes négatifs, qui correspondent a une époque ou les seropos ont partagés le virus entre eux et ces deux mots étaient synonyme de plombage, terrorisme, la mort etc...si vous voulez casser les stéréotypes, arrêtez d'utiliser ces mots. Parle de la prévention, des effets positifs, et surtout de la responsabilité ! Je suis passionné au sujet de la prep, mais elle n'est pas une laisser passer pour faire n'importe quoi.

\section{$[\ldots]$}

INTE010 Affiche qui ferait beaucoup polémique... Je pense qu'en France, à ce stade elle serait contre productive et pousserait certains décideurs vers plus de radicalité contre la Prep, elle serait incomprise... Cependant si rien ne bouge en 2016 faudra y repenser....

INTE001 Je suis d'accord INTE010. La PREP nous apprend aussi une certaine douceur.... Et pour être plus précis je crois que nous sommes tous conscients que nous ne pratiquons pas du sexe brut du bbk. Ce n'est pas ça la PREP

\section{[...]}

INTE011 Je ne suis pas sûr de comprendre votre discussion. Le "nous " c'est qui ? Tous les prepeurs ? Le " sexe brut du bbk " ça veut dire quoi ? Y aurait-il des bons barebackers et des mauvais barebackers? Dans ce cas, quels sont les critères d'admission au sexe sans latex vertueux ? Je suis un peu perdu... 
INTE001 On ne doit pas oublier - moi le premier - qu'au delà de la communication externe (auprès des autorités de santé, auprès des incrédules qui nous taxent d'irresponsables, auprès des financeurs qui rétorquent que nous allons dilapider l'argent public, auprès des moralisateurs qui nous traitent de Truvadawhores...), qu'au-delà de tout cela, il y a des personnes, il y a des situations. Il ne s'agit pas ici de juger. Néanmoins, INTE011, même si j'ai mis le " nous " là où j'aurais dû mettre le " je ", je réitère simplement que sous PREP, **je ${ }^{* *}$ ne me considère pas comme pratiquant du bbk. Peut-être as-tu, ou d'autres, une position différente, et effectivement, ce serait bien d'en débattre/témoigner... Je n'émets, par ailleurs, je pense pas de jugement sur le bbk je suis bien placé pour savoir qu'on a la sexualité qu'on peut/veut.

INTE011 Intéressant. En effet, la définition du bareback est polysémique et éminemment subjective. Les articles de (nom d'un sociologue spécialiste de la lutte contre le $\mathrm{VIH} / \mathrm{sida}$ ) sur la question, très accessibles, méritent d'être partagés.

\section{[...]}

La discussion ci-dessus a trait à la pertinence d'un message de promotion de la PrEP utilisé dans une campagne en Australie. Au début de celle-ci, les participants s'accordent pour dire qu'il serait « risqué » de tenir ce discours alors que les pouvoirs publics ne se sont pas encore positionnés sur l'accès à la PrEP. En effet, le « bareback » est connoté négativement, comme l'explique un des participants. Il renvoie à la peur du VIH et surtout du sida - et donc de la mort, comme au début de l'épidémie, ce dont témoignent les termes employés par INTE001 (" nous risquons de passer pour des terroristes ») et INTE003 (" plombage », " terrorisme », " la mort »). Il y a donc une volonté de différencier l'utilisation de la PrEP et le bareback. La PrEP « libère » de l'injonction à l'utilisation du préservatif car elle protège du VIH mais il ne faut pas pour autant qu'elle incite à ne plus en utiliser, à « faire n'importe quoi ». Cette expression, qui exprime un jugement de valeur, est récurrente dans les discussions au sein de PrEP'Dial. Elle est liée à la crainte d'une désinhibition des comportements sexuels et d'un relâchement de la prévention par l'abandon du préservatif. Un des participants, INTE011, souligne le danger de stigmatisation qui découle des propos tenus dans la discussion en suggérant qu'ils supposent l'existence de pratiques sans préservatif qui seraient acceptables et d'autres qui le seraient moins. L'utilisation de l'adjectif « vertueux » témoigne de la coloration morale de ces discours, qui n'est autre que le résultat de l'intériorisation d'une norme (BERGER et LUCKMAN, 2012 (1966)). En effet, les années de lutte contre le VIH/sida ont, d'une part, mené à l'institutionnalisation de l'utilisation du préservatif comme norme de prévention et, d'autre part, à une injonction au contrôle de leur sexualité par les individus. Le sida, apparu au moment où les homosexuels commençaient à obtenir la reconnaissance de leurs droits, renvoie à une sexualité tabou, déviante et dangereuse dans un contexte épidémiologique marqué par une forte prévalence de l'épidémie au sein d'une population marginalisée pour son orientation sexuelle. L'interaction avec INTE011, activiste de la lutte contre le VIH/sida, permet à INTE001 de justifier et d'expliciter son témoignage en se défendant de tout jugement concernant les pratiques sexuelles. Son discours est pris dans deux sortes de cadres (GOFFMAN, 1991), dont il semble s'accommoder au vu de son dernier commentaire. Un premier cadre, primaire, qui correspond à l'expérience de la sexualité qu'a cet individu, participant à l'essai ANRS Ipergay dont l'inclusion exigeait des pratiques sexuelles à risque. Un deuxième cadre, transformé, qui correspond à son expérience de la société, qui témoigne de sa connaissance des normes et de ce qu'il est possible de dire sur la PrEP dans un contexte d'incertitude de sa 
reconnaissance par les pouvoirs publics. La réponse de INTE011, sans approuver ou contredire les propos de INTE001, invite alors à la lecture de travaux scientifiques sur les différents sens que recouvre la définition du bareback. Par cette action, il propose des ressources validées socialement qui pourront permettre à celui qui s'y intéresse de s' " émanciper » des normes morales pesant sur la caractérisation de l'individu qui n'utilise pas de préservatif (BECKER, 1985, p. 97). De l'analyse de cet extrait émerge la figure sociale du « prepeur raisonnable », qui agit dans la mesure et qui est capable de maîtriser ses impulsions, conformément à la norme de prévention.

\section{Pendant la RTU : le « bon prepeur » et le « mauvais prepeur »}

\section{Encadré 2 - Extrait de PrEP'Dial, janvier 2016}

DISC086 [...] (Il explique qu'il va témoigner sur le déroulement de son premier rendez-vous à l'hôpital pour la PrEP). Le rendez-vous s'est bien passé, la condition pour avoir la prep est d'avoir eu des rapports non protégés avec au moins deux partenaires dans les six derniers mois. J'étais assez déterminé, j'ai expliqué qu'il $y$ avait beaucoup de " jeux " sans capotes avec mes partenaires et que j'avais déjà fait un tpe parce que mon pote avait pas prévenu qu'il allait jouir. [...] J'espère aussi pouvoir laisser mes partenaires jouir sans stresser grâce à la prep (c'est toujours difficile de profiter quand t'as peur en même temps que le mec se retire pas) et je pense continuer à utiliser la capote avec les potes que ça dérange pas ou qui le souhaitent, en bref alterner capote/sans capote. [...] Un acteur porno ricain dit sur son blog qu'il a été malade une semaine mais qu'après ça allait, par contre il dit aussi qu'il y a un peu un stigma social (les mecs qui prennent la prep sont mal considérés). [...]

HYPE002 Rassure toi, associer les Prepeurs à des "Truvada whores " est en train de disparaître parce que ces personnes prennent soin de leur santé et de leurs partenaires. [...]
DISC081 Attends, en lisant tous les commentaires, on peut pas avoir le traitement Truvada si on pratique pas des actes sexuels à risque ? [...]

EPHE002 C'est le médecin qui est à même d'estimer les besoins de prep ou non, c'est pas non plus une solution anodine, elle devrait selon moi arriver en dernier recours ou dans des cas bien precis (couples serodifferents, allergie au latex...) cela reste personnel mais ne banalisons pas non plus la prise de truvada!

\section{[...]}

DISC081 Pourquoi tu penses qu'il faut pas banaliser le Truvada? Enfin je pense que c'est un choix personnel, quiconque veut se protéger de cette manière doit pouvoir prendre ce traitement je trouve. Après prendre Truvada pour moi n'est pas une raison de pratiquer le sexe sans protection. Si je le prenais, ça serait plus pour diminuer le risque au maximum.

DISC086 [...] après avoir été bénévole pendant un an à aides (et eu des dizaines d'entretiens avec des mecs quand je faisais les actions en saunas) je crois pouvoir dire que la capote ne marche définitivement pas pour tout le monde, puisque des mecs continuent d'avoir des rapports non protégés. Je suis d'accord, être sous traitement, ce n'est pas anodin, mais je suis pragmatique, de toutes évidences, prendre une pilule est plus facile pour tout le monde. Je crois qu'il y a une difficulté à se rendre compte de ses privilèges aussi : quand on a pas de difficultés avec la capote, on a l'impression que les autres font juste preuve de mauvaise volonté. À la base, j'avais pas de problème avec la capote, et puis après une chirurgie, mon anus a rétréci/est devenu hypersensible, au point que le lube sur les condoms me brûlait. Ce n'est qu'au bout de deux ans que j'ai repris des grosses bites, quand un de mes partenaires a commencé à me prendre sans capote [...]

DISC086 Et je n'ai pas abordé la notion de plaisir. J'ai la " chance " d'avoir eu des problèmes de santé qui rendent mon utilisation de la prep plutôt vertueuse. Mais (et là je sors du retour d'expérience, c'est juste mon opinion personnelle) un mec qui voudrait la prep juste pour pratiquer le bareback serait tout autant légitime à mes yeux : parce qu'être enculé, ça me rend 
heureux, et peut-être que faire revenir le sperme dans nos jeux sexuels, c'est aussi quelque chose qui nous permet de nous sentir mieux.

DISC081 [...] EPHE002 je sais pas ce que tu apporterai comme solution première, mais en prenant compte de la nature humaine et le besoin du plaisir, par exemple moi je pratique pas sodomie sans protection mais je pratique pas la fellation avec capote non plus, donc je pense pour ceux qui veulent, Truvada c'est une bonne solution actuellement. Et encore une fois je souligne que c'est pas pour moi une raison de baiser n'importe comment avec n'importe qui, mais effectivement pouvoir pratiquer le sexe sans capote pourrait être un bénéfice. [...]

\section{$[\ldots]$}

\section{Encadré 3 - Extrait de PrEP'Dial, avril 2016}

DISC140 Petite expérience hier avec un Docteur spécialiste des IST de l'hôpital (Nom de l'hôpital) à Paris. J'ai simplement posé la question de son avis sur la Prep. La réponse a été assez négative: "Nous (médecin) ne soutenons pas ce traitement ", "rien de plus sûr que le préservatif ", "suivi médical très lourd", " ceux qui prennent la Prep le fond dans l'objectif de ne plus utiliser le préservatif, donc risque important de contamination avec d'autres IST", " on la propose car imposé par la loi, mais on ne sait pas pour combien de temps ça ne va pas durer "... Précision, ce docteur ne connaît rien de mon parcours ou de ma sexualité. Juste une visite de routine et j'en ai profité pour me renseigner... Des commentaires, des expériences? Merci !

\section{[...]}

HYPE003 Idem à (nom d'un hôpital). Le médecin m'avait dit "Dans votre cas, et je ne vous apprends rien, vous allez un jour vous contaminer au $\mathrm{VIH}$, le mieux serait de vous faire tester tous les mois pour qu'on le sache au plus tôt. Ah, la PrEP ? Moi je n'y crois pas, on va avoir un service pour la prescrire, mais personnellement je suis contre, je ne leur adresserait personne ». Le médecin qui te préfère séropo...

[...]

\section{Encadré 4 - Extrait de PrEP'Dial, novembre 2016}

DISC150 Pas un coup de gueule, juste une incompréhension. Maintenant la Prep mise en place, 2 mecs sur 3 sous Prep indiquent rechercher $d u$ bareback, no capotes, jus en bouche, etc... C'est ce que je constate dans les dials sur les apps en tout cas. Si j'ai bien appris ma leçon, la Prep vient en complément de la capote, et en dernier rempart lors de accidents rares et « oublis». Pas en remplacement de la capote, pas comme pilule miracle anti VIH... Si j'ai bien compris l'enjeu, c'est que l'un des dangers que provoquerais le remplacement de la capote par la Prep, serait, entre autre, l'explosion prochaine des MST/ IST déjà en forte hausse. Exemple sur Hornet : mettez le \#prep et constastez le nombre de mecs qui indiquent \#BBK ou le jus en emoticon... Sans parlez de ceux qui n'indiquent rien mais ne s'en privent pas. Hier, un mec me répond que grâce à la Prep, il peut désormais baiser sans capote avec des séro+. [...] Ok... Juste une incompréhension face au détournement flagrant de la mise sur le marché de ce nouvel et formidable outil complémentaire. Et si ce détournement au profit du bareback profitait aux MST / IST, pourrions-nous voir un jour des politiques remettre en question sa prise en charge par l'Etat... je ne le souhaite pas évidemment. Mais aujourd'hui, force est de constater que beaucoup voient en la Prep la possibilité enfin de baiser avec qui on veut sans capote, avaler son jus en bouche, se faire remplir avec... Ça me fait halluciner. C'est ce qu'on vous a expliqué à vous lors de votre $r d v$ ? Parce que moi, le médecin ne m'a clairement pas vendu la Prep comme cela...

EPHE072 Moi je suis entièrement d'accord avec ce que tu dis c'est assez hallucinant et honnêtement ça fait peur... Et il n'y a pas que ceux sous prep il y a aussi ceux qui ne le sont pas encore sous prétexte que leur(s) partenaire(s) le sont ! Ça se développe de plus en plus un peu comme le sm qui se démocratise de plus en plus notamment chez les jeunes alors qu'ils n'y connaissent rien...

\section{[...]}

ASSI001 [...]En quoi le SM est mal mis à part que ce ne soit pas ton truc ? Ensuite pour etre tout à fait honnête il y avait déjà des prises de risques 
avant prep... C'est d'ailleurs pour ça qu'il a fallut prep, et aujourd'hui les mecs s'assument et assumer leur choix et leur responsabilités dans leur prévention... [...]

\section{[...]}

EPHE072 Ce que je disais c'est qu'en ce moment on voit tout et n'importe quoi sous prétexte que ya la prep sous prétexte que le monde évolue et qu'on peut tout se permettre...

\section{[...]}

Partant du principe que l'utilisation d'un médicament ne doit pas être anodine certains participants élaborent des critères d'accès à la PrEP construits autour de raisons « acceptables » de ne pas utiliser de préservatif, comme par exemple une allergie au latex (encadré 2). Pour d'autres, elle doit être le «dernier rempart lors de accidents rares et « oublis » " (encadré 4). Le vocabulaire employé par ce participant s'inscrit dans le registre de l'apocalypse, avec la prédiction de "l'explosion des MST/IST » et les « dangers » de cette «pilule miracle ». On note chez ce participant une volonté d'interpeler en suscitant la peur par un discours alarmiste qui rappelle les premières années de l'épidémie et certaines campagnes de prévention axées sur le dégoût et la peur (PEZERIL, 2012), avec notamment une certaine méfiance vis-à-vis des PVVIH. Par ailleurs, dans la même discussion, un participant approuve ces propos et associe le fait de ne pas utiliser de préservatif à l'inexpérience sexuelle des jeunes et leur attrait qu'il a constaté pour le sadomasochisme. Si son propos est décousu, il est cependant révélateur de la représentation de l'abandon du préservatif comme une pratique déviante. Les commentaires qui suivent mettent en avant le fait que toutes les pratiques décrites existaient déjà avant la PrEP. Cette dernière permet de réduire le risque et non pas de développer des pratiques à risque. Utiliser la PrEP permet d'assumer sa sexualité, quelles que soient ses pratiques, sans risquer d'acquérir le VIH. Ainsi, les discussions montrent les significations que donnent les utilisateurs à la PrEP : elle permet davantage de sérénité en éloignant le stress et la peur. De plus, selon DISC086 (encadré 2) l'utilisation du préservatif peut être contraignante et son abandon n'est pas le résultat de la mauvaise volonté de l'individu. Le récit de son expérience sur le terrain en tant que militant, dans un sauna gay, lui sert à valider son point de vue en livrant « une vision tout à la fois experte, subjective et singulière » (DENOUËL, 2011, p. 79) de la diversité des raisons de l'abandon du préservatif et de la diversité des manières d'utiliser la PrEP. Selon lui, les pratiques sexuelles doivent permettre de s'épanouir. Ces propos témoignent d'une interprétation de la sexualité qui va à l'encontre de la moralité, parce qu'elle convoque la notion de plaisir dans un cadre dominé par la peur du VIH. On observe également la mobilisation du médecin tantôt comme une figure d'autorité, tantôt comme un frein à l'accès à la PrEP. Ainsi, pour certains il est le seul juge des besoins en prévention de ses patients. Sa subjectivité est complètement reniée par le participant qui ne le voit plus que comme le grand détenteur de la vérité : ce qu'il affirme est incontestable, quand bien même il s'agirait de son point de vue (encadrés 2 et 4 ). Pour d'autres, le médecin doit être à l'écoute de ce qui convient le mieux au patient sans porter de jugement sur ses pratiques. Ainsi, des situations rapportées par des participants (encadré 3) montrent que certains médecins ne tiennent pas compte de la diversité des pratiques et des situations et perpétuent l'idée que le fait de ne pas utiliser de préservatif est une question de mauvaise volonté. Ainsi, il vont jusqu'à nier l'efficacité de la PrEP en proposant au patient un suivi dans l'attente de sa séroconversion au VIH. Ces situations sont rapportées de manière fréquente 
pendant la RTU, qui n'était en quelque sorte qu'un prolongement de l'essai ANRS Ipergay ; elles sont moindres à partir de l'AMM car l'efficacité de la PrEP n'est alors plus discutable. De ces discussions émergent les figures sociales du « bon prepeur » et du " mauvais prepeur » qui se construisent autour de la norme de l'utilisation du préservatif. Ainsi, pour qu'on l'autorise à déroger à la norme de l'utilisation du préservatif, le " prepeur » doit être exemplaire. Le " bon prepeur », c'est celui qui dit utiliser la PrEP avec une observance irréprochable et toujours avec des préservatifs, pour réduire le risque d'acquisition ou de transmission d'autres IST, dont la PrEP ne protège pas. Pour lui la PrEP est davantage une sécurité supplémentaire, au cas où il y aurait un « accident » - oubli, rupture - de préservatif. À l'autre extrémité de l'axe, le « mauvais prepeur » est celui qui multiplie les expériences et les partenaires, sans utiliser de préservatif. Or, utiliser la PrEP c'est reconnaître que l'on prend des risques. Mais utiliser la PrEP c'est aussi faire en sorte de pallier ces prises de risque en faisant le choix d'un moyen de prévention au schéma d'utilisation pourtant complexe dont l'observance est la clé de l'efficacité.

\section{Après l'annonce de l'AMM : le «prepeur responsable »}

\section{Encadré 5 - Extrait de PrEP'Dial, février 2017}

ASSI001 J'entends souvent des mecs qui s'interrogent sur La Prep et qui me disent : je sais pas, si je prend La prep j'ai peur de devenir une "Pute à jus ", "Salope qui ne se respecte pas", " super grosse chienne "... et j'en passe. -1- et alors ?! Tu fais ce que tu veux, des putes des Salopes et des chiennes il y en $a$, et il y en aura toujours, mais toujours des BONNES putes, Salopes ou chiennes. -2- il va falloir que les polices des mours comprennent enfin que leur jugements ne font pas avancer la prévention : "Support ! Don't Punish » Soyez des sacs à foutre si cela vous rend heureux, n'oubliez juste Pas de prendre soin de vous et de vos partenaires, le reste on s'en fou personne n'a à blâmer vos comportements et vos choix. Le principale : ASSUMONS NOS RESPONSABILITÉS

DISC358 Amen. De toute façon c'est pas parce qu'on est prepeur qu'on est obligé de changer sa sexualité, ca peut aider à être plus serein dans certaines pratiques après chacun fait ce qu'il veut

\section{[...]}

INTE012 La prévention n'a pas besoin de dames patronesses à la Boutin. La stigmatisation et la honte n'empêchent pas une contamination. Le bien être si.

[...]

EPHE073 moi on ma traité de pute à Truvada

DISC384 Oui moi On m'appelle trouvada c'est drôle et $\mathrm{Ca}$ me fait beaucoup rire. Surtout quand tu reçois des leçons de morale de gars qui critiquent la prep et son pas foutu d'aller faire un test de dépistage lol. La prévention $\mathrm{Ca}$ commence par le dépistage et tout le monde à Quand meme tendance à un peu oublier $\mathrm{Ca}$

$[\ldots]$

\section{Encadré 6 - Extrait de PrEP'Dial, juin 2017}

DISC497 Ça y est per jour, un gros changement dans ma sexualité après 25 ans de kpot (il publie la photo d'une boîte de Truvada)

DISC001 Bravo ! Bienvenu parmi les prepeurs!! Mais tu envisages d'abandonner la capote totalement?

INTE023 Enjoy bareback! Ne sois juste pas anti-capote (vis à vis de ceux qui veulent en mettre)

DISC497 Non je n'abandonne pas la kpot évidemment mais les 2 vont vivre ensemble

DISC001 Je revis Lol Parce qu'il y a 2-3 posts assez chauds en ce moment sur le sujet où les débats font rage Lol. Ton choix est sage et responsable et je salue ta décision. La pilule bleue s'accomode plutôt bien du latex. Mais je confirme qu'on se sent plus léger. 
HYPE003 Il n'y a pas un choix meilleur qu'un autre sur PrEP'Dial

DISC001 HYPE003 c'est ton avis, que je ne partage pas comme tu le sais

HYPE003 DISC001, c'est la charte du groupe. Alors si elle ne te convient pas, tu es libre de partir.

\section{[...]}

INTE021 Welcome to PrEPLAND. Ma sexualité s'est construite depuis mes "débuts " sous la coupe et le discours ambiant de "la kpote ou l'enfer » et ceci durant presque 30 ans... Je salue ta décision d'envisager la cohabitation de la PrEP et kpote... J ai commence y a un an la PrEP avec ces idéaux... imperceptiblement, le temps, des examens trimestriels bons et une confiance grandissante ont fait que...je délaisse de plus en plus la kpote... Je « vis » enfin sexuellement en compagnie de PrEP en continu. Quelque soit ton futur parcours, prends soins de toi et have fun.

DISC001 INTE021, je salue ton choix de faire cohabiter les 2 moyens de prévention. Cependant, je me dois de souligner que la Prep ne protège que du VIH et pas des autres IST. Dans mon cas, 1,5 mois avec une utilisation intermittente de la capote m'a offert la syphilis...

[...]

DISC500 C'est vrai que qd on a vécu depuis 40 ans avec la capote cest un sacré changement !!

AUTR007 Il faut voir la prep comme la pilule contraceptive des années 70 et la révolution sexuelle que cela a permis! Marre des moralisateurs !!! On fait des bilans tous les 3 mois plus que ceux qui font des dépistages tous les 2 ans et qui se font sucer sans capote et transmettent des IST !

ASSI013 PrEPeur depuis 1 an j'ai connu le temps béni d'avant le SIDA. La capote à toujours été pour moi un outil de protection bien inconfortable lorsqu'il a fallu se protéger. Et au fil du temps je l'ai de moins en moins utilisée - ou de plus en plus " oubliee ". Puis l'ai abandonnée. J'ai eu la chance de ne RIEN attrapé pendant toutes ces années sans protection (disons 20 ans). Alors quand la PrEP à été proposée je me suis renseigné j'ai pris rendez-vous et...le médecin rencontré n'a pas hésité 1 seconde pour me prescrire la pilule bleue. Juste pour dire que peut avoir des pratiques à risque... et être parfaitement conscient des conséquences possibles. Et tenter de les éviter.

On constate l'émergence d'un discours montrant une nécessité, pour que la prévention soit efficace, de dépasser l'opposition entre les " bons » et les « mauvais prepeurs » vers une conception de l'utilisation de la PrEP comme un acte responsable. La responsabilité désigne le fait de se reconnaître comme l'auteur de ses propres actes mais "c'est d'autrui plutôt que du for intérieur, que l'injonction morale est dite procéder [...] On devient responsable du dommage, parce que, d'abord, on est responsable d'autrui » (RICFUR, 1994, p. 43-44). Si l'on s'attache à cette définition, la responsabilité est entendue comme le fait de prendre soin de soi et de ses partenaires, ce que la PrEP permet, notamment avec les dépistages et le suivi régulier. De plus, la PrEP contribue au bonheur et à la sérénité dans la sexualité, ce qui participe au bien-être de l'individu, principe énoncé dans le Préambule à la Constitution de l'Organisation mondiale de la santé (OMS)7. L'utilisation de la PrEP est qualifiée de " gros changement » (encadré 6) dans la sexualité des participants car elle met à distance l'injonction à l'utilisation du préservatif qui était subie et entrainait la stigmatisation des individus qui ne s'y soumettaient pas. Certains commentaires s'inquiètent des risques d'IST et prônent l'utilisation combinée du préservatif comme meilleure prévention. Ces participants se basent sur leur expérience de découverte d'une IST pour ne pas avoir utilisé de préservatif avec la PrEP.

7 Préambule à la Constitution de l'Organisation mondiale de la Santé, tel qu'adopté par la Conférence internationale sur la Santé, New York, 19 juin -22 juillet 1946; signé le 22 juillet 1946 par les représentants de 61 Etats. (Actes officiels de l'Organisation mondiale de la Santé, $\mathrm{n}^{\circ} .2$, p. 100) et entré en vigueur le 7 avril 1948. 
Par exemple pour DISC001 (encadré 6), il n'y a qu'une seule manière d'être responsable, c'est d'utiliser la PrEP en plus du préservatif. Il est rappelé à l'ordre par HYPE003 pour manquement à la charte du groupe. On note donc ici un exemple du cadrage des discours par le dispositif et l'intériorisation des règles de fonctionnement par les participants. Enfin, l'intervention de INTE021 concernant la construction de sa sexualité autour de la norme de l'utilisation du préservatif montre bien que l'intériorisation de l'injonction au préservatif pouvait se faire en contradiction avec les capacités de l'individu à l'utiliser. Celui-ci entre alors dans un comportement déviant lorsqu'il ne parvient pas à la suivre. Si l'on constate la persistance de l'injonction à l'utilisation du préservatif, par exemple lorsque DISC497 (encadré 6) dit « Non je n'abandonne pas la kpot évidemment », les interactions avec des participants plus expérimentés comme INTE021 témoignent d'un apprentissage de la déviance. En effet, le récit de ce dernier montre que le choix de prévention ne doit pas se faire en fonction d'une norme pouvant être difficile à appliquer, mais qu'il se construit par l'expérience. Selon H. S. Becker (1985 : 38), la déviance est une propriété « de l'interaction entre la personne qui commet l'acte et celles qui réagissent à cet acte ». Pour lui, c'est par la sociabilisation et la réalisation de nouvelles expériences avec des déviants plus expérimentés que l'individu entre dans un mode de comportement déviant. Au sein de PrEP'Dial, chaque individu peut apprendre, par le partage d'expériences, les interactions avec les autres individus, à participer à certaines activités considérées comme déviantes par d'autres.

\section{Conclusion}

L'objectif premier de cette contribution a été d'approcher une forme narrative particulière, le témoignage en ligne, comme objet d'analyse des significations attachées à l'utilisation de la PrEP. L'analyse des récits de soi, en tant qu'objets contraints par un dispositif sociotechnique institutionnalisé et mouvants dans l'interaction, s'avère pertinente dans la mesure où elle a permis d'observer les variations dans la dimension normative attachée à la PrEP. De plus, l'appui sur les travaux de H. S. Becker a permis de mettre en évidence des figures sociales de l'usager de la PrEP, qui se constituent par le croisement de la nature du comportement (conforme ou non-conforme à la norme) avec les réactions qu'il suscite (c'est-à-dire en fonction de la perception qu'en ont les participants au groupe) (BECKER, 1985, p. 43-44), dans une approche séquentielle. Ainsi, ont émergé la figure du " prepeur raisonnable », celle du « bon prepeur » en opposition à la figure du " mauvais prepeur », puis celle du " prepeur responsable ». On a constaté que la construction et l'évolution de ces figures sont dépendantes d'un contexte politique relatif à l'autorisation de la PrEP et à sa prise en charge par l'État. L'analyse des témoignages et de leur dimension interactive a également mis en évidence un certain apprentissage de la déviance, dont il serait intéressant de prolonger l'analyse notamment par le concept de carrière, qui " désigne les facteurs dont dépend la mobilité d'une position à une autre, c'est-à-dire aussi bien les faits objectifs relevant de la structure sociale que les changements dans les perspectives, les motivations et les désirs de l'individu » (BECKER, 1985, p. 47). Cela permettrait de caractériser les justifications menant un individu à s'approprier les messages de prévention, l'appropriation étant entendue comme " l'intégration du message dans un cadre de vie quotidien, quelle qu'en soit la forme, du changement de comportement incité par le message à l'ignorance délibérée du message » (KIVITS et al., 2014, p. 156). 


\section{Bibliographie}

AKRICH, Madeleine. Les listes de discussion comme communautés en ligne: outils de description et méthodes d'analyse. Papiers de recherche du CSI, n. 25, p.1-101, 2012.

AKRICH, Madeleine; MEADEL, Cécile. Les échanges entre patients sur l'internet, La presse médicale, $v$. 38, n. 10, p. 1484-1493, 2009.

AKRICH, Madeleine; MEADEL, Cécile. De l'interaction à l'engagement: les collectifs électroniques, nouveaux militants de la santé. Hermès, La Revue, v. 1, n. 47, p. 145-153, 2007.

BADOUARD, Romain; MABI, Clément; MONNOYER-SMITH, Laurence. Le débat et ses arènes: à propos de la matérialité des espaces de discussion. Questions de communication, n. 30, p. 7-23, 2016.

BECKER, Howard S. Outsiders: études de sociologie de la déviance. Paris: Métailié, 1985.

BERGER, Peter; LUCKMAN, Thomas. La construction sociale de la réalité. Paris: Armand Colin, 2012.

CARBOU, Guillaume. Presse en ligne et communautés cognitives: les sections commentaires d'Atlantico.fr et de l'Humanité.fr durant "l'affaire DSK". Réseaux, n. 193, p. 193-223, 2015.

CEFAï, Daniel. Publics, problèmes publics, arènes publiques... Questions de communication, n. 30, p. 25-64, 2016.

CORDONNIER, Sarah. (Dir.). Trajectoire et témoignage: pour une réflexion pluridisciplinaire. France: Éditions des Archives Contemporaines, 2015.

DENOUËL, Julie. Production participative d'information sur internet: expression citoyenne, engagement civique et culture de soi. Recherches en Communication, n. 36, p. 71-84, 2011.

DODIER, Nicolas. L'espace public de la recherche médicale. Autour de l'affaire de la ciclosporine, Réseaux. Communication, technologie, société, $n^{\circ} 95$, p. 107-154, 1999.

FLICHY, Patrice. L'imaginaire d'internet. Paris: La
Découverte, 2001.

GOFFMAN, Erving. Les rites d'interaction. Paris: Éditions de Minuit, 1974.

GOFFMAN, Erving. Façons de parler. Paris: Éditions de Minuit, 1987.

GOFFMAN, Erving. Les cadres de l'expérience. Paris: Éditions de Minuit, 1991.

GRANJON, Fabien. Reconnaissance et usages d'internet: une sociologie critique des pratiques informatiques connectées. Paris: Éditions Transvalor; Presses des Mines, 2012.

GIRARD, Gabriel. Les réseaux mis en contexte: une perspective comparative de la prise en charge du VIH en France et au Québec. In: BROSSARD. Baptiste; WHITE, Deena. (Dir.). La santé en réseaux: explorations des approches relationnelles dans la recherche sociale au Québec. Québec: Presses de l'Université du Québec, 2016. p. 169-190.

GIRARD, Gabriel. Les homosexuels et le risque du sida: individu, communauté et prévention. Rennes: Presses Universitaires de Rennes, 2013.

HINE, Christine. Ethnography for the internet: embedded, embodied and everyday. London: Bloomsbury, 2015.

JOUËT, Josiane; LE CAROFF, Coralie. L'observation ethnographique en ligne. In: BARATS, Christine. (Dir.). Manuel d'analyse du web. Paris: Armand Colin, 2013. p. 147-165.

KIVITS, Joëlle et al. L'appropriation de l'information médiatique au sujet de la prévention et du dépistage des cancers. Le Temps des Médias, v. 2, n. 23, p. 151-163, 2014.

LE BRETON, David. L'interactionnisme symbolique. Paris: PUF, 2016.

LE CAROFF, Coralie. Le genre et la prise de parole politique sur facebook. Participations, v. 2, n. 12, p. 109-137, 2015.

MARCOCCIA, Michel. Analyser la communication nu- 
mérique écrite. Paris: Armand Colin, 2016.

MEAD, George H. L'esprit, le soi et la société. Paris: PUF, 1963.

$M E H L$, Dominique. Le témoin, figure emblématique de l'espace privé/public. In: CEFAï, Daniel; PASQUIER, Dominique. (Dir.). Les sens du public: publics politiques, publics médiatiques. Paris: PUF, 2003. p. 489-502.

NIEWIADOMSKI, Christophe; DELORY-MOMBERGER, Christine. (Dir.). La mise en récit de soi: place de la recherche biographique dans les sciences humaines et sociales. Villeneuve-d'Ascq: Presses Universitaires du Septentrion, 2013.

PAGANELLI, Céline; CLAVIER, Viviane. Le forum de discussion: une ressource informationnelle hybride entre information grand public et information spécialisée. In: YASRI-LABRIQUE, Eléonore. (Dir.). Les forums de discussion: agoras du XXIè siècle? Théories, enjeux et pratiques discursives. Paris: L'Harmattan, 2011. p. 39-54.

PASTINELLI, Madeleine. Pour en finir avec l'ethnographie du virtuel! Des enjeux méthodologiques de l'enquête de terrain en ligne. Anthropologie et Sociétés, v. 35, n. 1-2, p. 35-52, 2011.
PEZERIL, Charlotte. Le dégoût dans les campagnes de lutte contre le sida. Ethnologie française, $v$. 41, p. 79-88, 2011.

RICCEUR, Paul. Le concept de responsabilité: essai d'analyse sémantique. Revue Esprit, p. 28-48, 1994.

ROJAS CASTRO, Daniela; LE GALL, Jean-Marie; JABLONSKI, Olivier. Améliorer la santé des personnes séropositives au VIH: seronet, un nouvel outil de l'approche communautaire, Santé publique, Hors-série, p. 129-141, 2009.

TRACHMAN, Mathieu; GIRARD, Gabriel. Le ciblage des hommes faillibles: stratégies de communication et enjeux moraux dans un essai de prophylaxie préexposition. In: OLLIVIER-YANIV, Caroline; COUDERC, Mathilde. (Dir.). Recrutement-engagement dans des essais cliniques en prévention: contextes, logiques sociales et médiations. Paris: ANRS, 2018. p. 21-35.

VELTER, Annie et al. Comportements sexuels entre hommes à l'ère de la prévention combinée: résultats de l'enquête presse gays et lesbiennes 2011. Bulletin Epidémiologique Hebdomadaire, n. 39-40, p. 510-516, 2013.

Recebido em : 12/03/2019

Aprovado em : 18/05/2019

Cécile Loriato est doctorante en Sciences de l'information et de la communication (Section CNU: 71), Céditec - Centre d'Étude des Discours, Images, Textes, Écrits, Communications, Université Paris-Est Créteil Val-de-Marne. e-mail: cecile. loriato@gmail.com

61, avenue du Général de Gaulle, 94010 Créteil Cédex - Tel : 0633808559 